\title{
Phase Noise of an HTS Resonator Operated in the Nonlinear Regime
}

\author{
Svetlana A. Vitusevich, Norbert Klein, Alexander G. Zaitsev, and Jochen Geerk
}

\begin{abstract}
Nonlinear properties of high-temperature superconducting (HTS) strongly coupled resonators at different input power have been investigated by measurements of the quality factor as a function of input power as well as by generation of the intermodulation distortion (IMD). The double-sided YBCO films on $\mathrm{CeO}_{2}$ buffered sapphire demonstrate advanced performance with the microwave surface resistance of $\sim 0.3 \mathrm{~m} \Omega$ at $8.5 \mathrm{GHz}$ at $77 \mathrm{~K}$ and an IMD third-order interception point estimated at $P_{\text {circ }} \approx$ $70 \mathrm{~W}$. A parallel feedback oscillator was assembled using a transmission type HTS resonator cooled with liquid nitrogen and a room temperature low noise amplifier. Results on the phase noise investigation of the $2.3 \mathrm{GHz}$ oscillator based on HTS resonator operated in the nonlinear regime demonstrated a deviation from the simple model of the phase noise, which predicts an up-conversion of $1 / f$ noise of the amplifier to oscillator phase noise. The later can be explained by the phase noise introduced additionally due to the nonlinear response of the HTS resonator.
\end{abstract}

Index Terms-Intermodulation, oscillator noise, phase noise, resonator, superconductor.

\section{INTRODUCTION}

H IGH-TEMPERATURE superconducting (HTS) thin films are used for designing high quality resonators and oscillators for satellite communications due to extremely low microwave surface resistance and low losses in these materials [1]. Nonlinear effects in a HTS resonator can result in changes in parameters of microwave telecommunication systems based on such resonators. Intermodulation distortion (IMD) is a serious problem, since it can result in mixing of signals leading to the additional noise sources. The phase noise in an oscillator is strongly dependent on circuit components, especially on the resonator's unloaded quality factor, $Q_{U}$, and the coupling ports factors [2]. Both factors are affected by the microwave surface impedance of the HTS film and may result in increasing noise level of the oscillator. The microwave surface impedance of the HTS film is strongly dependent on the microwave power level, especially when the superconducting resonator is included in the oscillator circuit containing an amplifier working in the strong compression and nonlinear regime. Additionally,

Manuscript received August 6, 2002.

S. A. Vitusevich is with Forschungszentrum Jülich, Institut für Schichten und Grenzflächen, D-52425 Jülich, Germany, and also with the National Academy of Science of Ukraine, Institute of Semiconductor Physics, Kiev, Ukraine (e-mail: s.vitusevich@ fz-juelich.de).

N. Klein is with Forschungszentrum Jülich, Institut für Schichten und Grenzflächen, D-52425 Jülich, Germany (e-mail: n klein@fz-juelich.de).

A. G. Zaitsev is with Forschungszentrum Karlsruhe, Institut für Festkörperphysik, D-76021 Karlsruhe, Germany (e-mail: alexander.zaitsev@ifp.fzk.de).

J. Geerk is with Forschungszentrum Karlsruhe, Institut für Festkörperhysik,

D-76021 Karlsruhe, Germany (e-mail: jochen.geerk@ifp.fzk.de).

Digital Object Identifier 10.1109/TASC.2003.813720 the coupling of the HTS resonator will be affected by the steady-state power conditions in the free running oscillator circuit. In a feedback-oscillator the output signal of a low $1 / f$ - noise microwave amplifier is passed through a two-port microwave resonator and fed back to the amplifier input. If the total phase shift around the oscillator loop is an integer of $2 \pi$ and the total loop amplification (equal to amplifier gain minus the total insertion loss of the resonator and all other components) exceeds unity, this device oscillates at the resonant frequency of the resonator. It is known, that there is a trade-off between the output power and the phase noise level of such an oscillator. The phase noise in the resonator circuit is minimized under the optimal relation between the loaded and unloaded quality factors of $Q_{L}=Q_{U} / 2$ [3], which is usually established by the coupling port conditions. However, in the case of the HTS resonator these conditions are dependent on the microwave power. Consequently, the accurate measurements of the phase fluctuations in the amplifier and oscillator are important for understanding the nature of the noise sources in these devices and the origin of the noise up-conversion.

In this paper the intrinsic nonlinearities in the HTS hairpin resonator at different input microwave levels have been investigated and the phase noise measurements have been used to determine the noise sources of the feedback oscillator assembled on the basis of a transmission type HTS resonator working in the nonlinear regime.

\section{EXPERIMENTAL DETAILS}

\section{A. Resonator}

Epitaxial (001) YBCO $\left(\mathrm{YBa}_{2} \mathrm{Cu}_{3} \mathrm{O}_{7-x}\right)$ films were prepared on both sides of (001) $\mathrm{CeO}_{2}$ buffered $r$-cut sapphire wafers by sputtering using the simultaneous double-sided deposition (ASIDOD) technique [4]. The sapphire wafers were 3 inches in diameter and $0.43 \mathrm{~mm}$ thick. The thickness of the $\mathrm{CeO}_{2}$ buffer layers was about $20 \mathrm{~nm}$. For the present work we used $400 \mathrm{~nm}$ thick YBCO films, which exhibited critical temperatures above $90 \mathrm{~K}$ and self-field critical current densities $J_{c}(77 \mathrm{~K}) \geq 3 \mathrm{MA} / \mathrm{cm}^{2}$ homogeneously distributed over the entire film surfaces. The critical parameters were measured inductively. The microwave surface resistance, $R_{S}$, of the unpatterned films (measured at $8.5 \mathrm{GHz}$ using a dielectric resonator technique [4]) was typically $\sim 0.3 \mathrm{~m} \Omega$ at $77 \mathrm{~K}$ on both sides of the wafers. The power handling capability was characterized by a breakdown hf field above $10 \mathrm{mT}$.

The microstrip half-wavelength resonators were fabricated using the "hairpin"-like layout shown in the insert of Fig. 1. The details of patterning and contacting the YBCO films are reported 


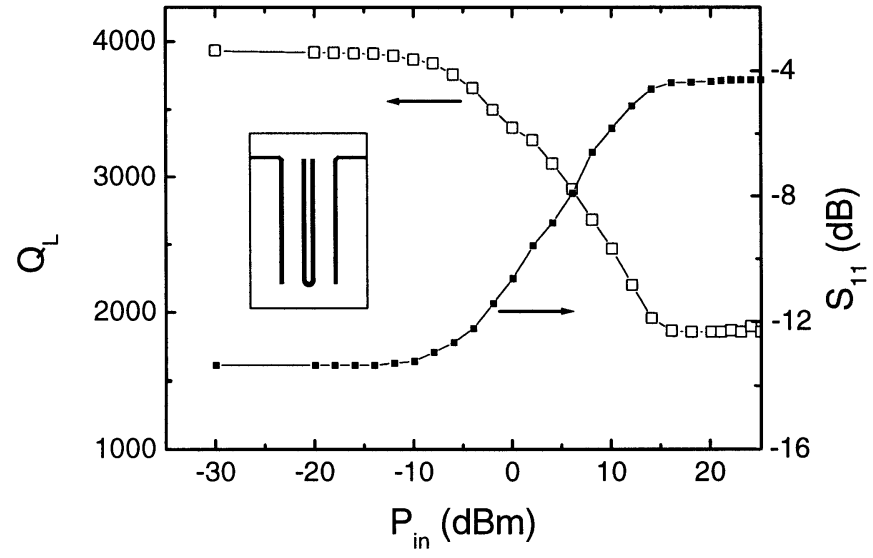

Fig. 1. Loaded quality factor $Q_{L}$ and the reflection coefficient $\mathrm{S} 11(\mathrm{~dB})$ vs the input microwave power measured for a YBCO microstrip resonator at $T=78 \mathrm{~K}$ and $f_{0} \approx 2.3 \mathrm{GHz}$. The resonator layout is shown in the inset.

in [5]. The width of the lines of $0.41 \mathrm{~mm}$ corresponded to $50 \Omega$ wave impedance on the $0.43 \mathrm{~mm}$ thick sapphire substrate. The length of the resonator of $27.2 \mathrm{~mm}$ yielded a fundamental frequency $f_{0} \approx 2.3 \mathrm{GHz}$.

\section{B. Intermodulation Distortion Measurement}

The resonator was strongly coupled in order to obtain a wide passband suitable for the measurements of high-order IMD products far from the frequency $f_{0}$. The IMD measurements were performed using a standard scheme [6] with two tones of equal power, one at the frequency $f_{1}$ and the other at $f_{2}$ with $\left(f_{2}-f_{1}\right)=20 \mathrm{kHz}$. The input signals were produced by two HP E4422B generators. The response was measured with an Anritsu MS2665C signal analyzer. The conventional frequency dependent measurements of the transmission, $S_{21}$, and reflection, $S_{11}$, parameters were performed using an HP8720C network analyzer. From these measurements the loaded quality factor of the resonator, $Q_{L}$, was determined. In the linear regime $Q_{L}$ was obtained from the $3 \mathrm{~dB}$ bandwidth, whereas in the nonlinear regime it was obtained from the transmission at the maximum of the resonance curve [6].

\section{Phase Noise Measurement of Amplifier}

The amplifier phase noise was measured in a two branch configuration employing a HP E5500 phase noise measurement system. A vector network analyzer is used as a source in continuous wave mode to provide a single signal at $2.3 \mathrm{GHz}$. A CW signal generated by a HP 83640A synthesized sweeper is split into two equal parts, one passing through a phase shifter, the other through the amplifier. When the phase difference is adjusted to 90 degree (quadrature), the output voltage is equal to zero. Therefore any phase fluctuation of the amplifier was registered as a voltage fluctuation at the output.

\section{Phase Noise Measurement of $2.3 \mathrm{GHz}$ Oscillator}

We have designed and investigated an oscillator consisting of a HTS hairpin resonator, a low noise HEMT amplifier (MITEQ model N AFS1), and a phase shifter. The amplifier operates at room temperature. Both coupling antennas were strongly cou-

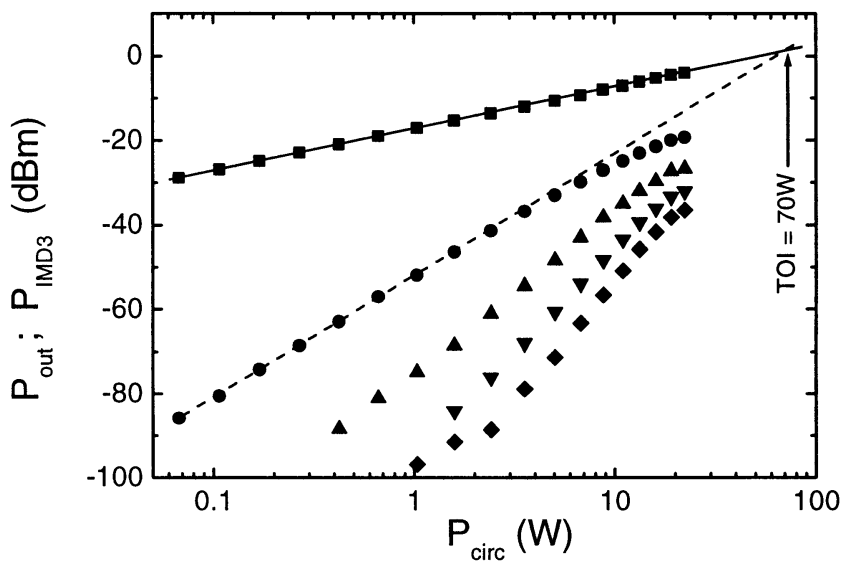

Fig. 2. Measured output power in the fundamental and intermodulation signals in a YBCO microstrip resonator on buffered sapphire. The symbols stand for $(\square)$

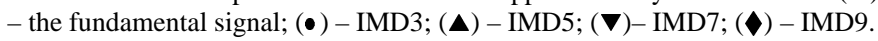

pled, such that the resonator insertion loss was about $6 \mathrm{~dB}$ at power $12 \mathrm{dBm}$.

Phase noise measurements of the oscillator are performed in a phase-locked loop configuration with using a low noise down converter with noise level well below the measured noise in the oscillator overall the frequency range investigated.

\section{IMD MEASUREMENT RESULTS}

The IMD measurements were performed in a wide temperature range between $4.2 \mathrm{~K}$ and $85 \mathrm{~K}$. However, in this brief report we focus only on the measurements at liquid nitrogen temperature, since the results obtained at other temperatures led to the same conclusions.

At $78 \mathrm{~K}$ the microstrip resonator exhibited a linear response up to an input power $P_{\text {in }}=-5 \mathrm{dBm}$, which resulted in a circulating power of $P_{\text {circ }} \approx 0.5 \mathrm{~W}$. In the linear regime the $Q_{L}$ was 3975 (see Fig. 1), which corresponds to an unloaded quality factor $Q_{0} \approx 35000$. The resonance curves of the transmitted power, $P_{\text {out }}$, versus frequency exhibited a regular Lorentzian shape [6]. With increasing power above $-5 \mathrm{dBm}, Q_{L}$ decreased and the resonance curves became deformed, $f_{0}$ shifted downwards and the coupling of the resonator became weaker. Such a combination of nonlinear effects was earlier reported for the YBCO stripline [7] and coplanar [8] resonators on $\mathrm{LaAlO}_{3}$ substrates. To illustrate this nonlinear behavior, we plotted in Fig. 1 typical dependences of $Q_{L}$ and $S_{11}$ versus the microwave power which are the most relevant for the present work.

The nonlinear response of the YBCO resonators was characterized by strong intermodulation distortion. In the two-tone measurements with the signal frequencies $f_{1}$ and $f_{2}$ (with $f_{0}-f_{1}=f_{2}-f_{0}=\delta f$ ) the third-order IMD product (IMD3) appears at frequencies of $f_{0} \pm 3 \delta f$, the fifth-order one (IMD5) at $f_{0} \pm 5 \delta f$, etc. The IMD signals exhibited continuous growth with the enhancement of the circulating power in the resonator (see Fig. 2). Up to $P_{\text {circ }}$ of approximately $5 \mathrm{~mW}$ the power $P_{\text {IMD3 }}$ was proportional to $P_{\text {circ }}{ }^{3}$, which is often reported for HTS resonators [6]. However, in the high power range a lower slope was observed. By approximating the dependence $P_{\mathrm{IMD} 3} \propto P_{\text {circ }}{ }^{3}$ toward higher power, the third order interception (TOI) point was estimated at $P_{\text {circ }} \approx 70 \mathrm{~W}$. The obtained 


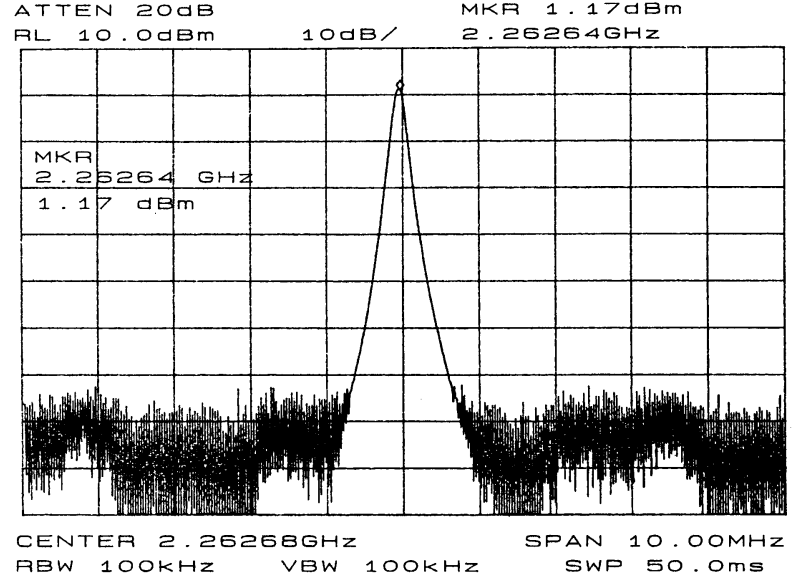

Fig. 3. Output power spectrum of the 2.3-GHz free running oscillator based on an HTS resonator.

results on measurement of the quality factor as a function of input power as well as on generation of intermodulation distortion were used for the phase noise investigation and analysis.

\section{OSCILLATOR RESULTS}

The $2.3 \mathrm{GHz}$ oscillators were designed in a parallel feedback configuration. The signal from HTS resonator is fed back to the input of room temperature amplifier and passes through a coupler with $10 \mathrm{~dB}$ output and the phase shifter, which enables adjustment of the phase around the oscillator loop to meet the oscillation condition.

The microwave power passed through the HTS resonator was found to be $12 \mathrm{dBm}$. The resonator coupling measured at this power was about 0.41 and 0.44 for port 1 and port 2 , respectively, resulting in a loaded $Q$-value above 2200 .

The unloaded $Q_{U}$ at the power $12 \mathrm{dBm}$ is calculated to be 4076. The value is approximately double of loaded $Q_{L}$. According to [3] this is optimal ratio for minimal noise level in the oscillator.

The performance of the oscillator was tested using a spectrum analyzer and the E5500 phase noise E5500 measurement set up. Fig. 3 shows the power spectrum of the oscillator. The oscillation frequency is $2.26 \mathrm{GHz}$ at $78 \mathrm{~K}$. An output power of $1.17 \mathrm{dBm}$ was measured at this frequency.

The phase noise measurement results for the amplifier and oscillator are shown in Fig. 4. The amplifier phase noise exhibits an almost ideal $1 / f$ dependence without generation-recombination component with absolute value of $-140 \mathrm{dBc} / \mathrm{Hz}$ at frequency offset of $1 \mathrm{kHz}$, which is nearly independent on the value of the voltage applied to the transistor. Curve 2 in the figure shows the level of the measured oscillator phase noise.

The basic phase noise behavior of the oscillator circuit is described by the Leeson model [9], which is based on the properties of the oscillator components. Phase noise of the amplifier is transformed into oscillator phase noise according to the formula:

$$
L_{o s c}=10 \cdot \log \left[1+\frac{f_{0}^{2}}{4 Q_{L}^{2} f_{m}^{2}}\right]+10 \cdot \log \left[\frac{\alpha}{f_{m}}+\frac{G F k T}{P}\right] .
$$

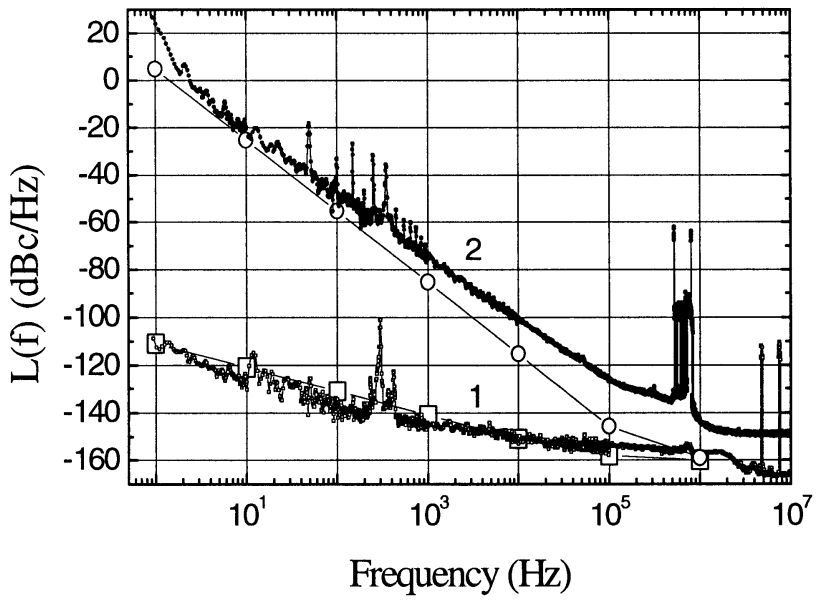

Fig. 4. Measured single side band phase noise of (1) the amplifier and of (2) the oscillator at frequency $2.26 \mathrm{GHz}$. The squares is a $1 / f$ fit to the amplifier noise, the circles is the oscillator phase noise calculated from the fit to the amplifier phase noise according to the Leeson formula.

The second term in (1) represents the amplifier noise consisting of the white noise determined by the amplifier gain $G$, noise figure $F$, physical temperature $T$ and output power $P$. In addition, there is $1 / f$ noise leading to a fluctuation amplitude $\alpha / f_{m}$ at the frequency distanced by $\pm f_{m}$ from the frequency $f_{0}$. The first term in (1) represents the resonator noise amplification factor, which is equal to $3 \mathrm{~dB}$ for $f_{m}=1 / 2 \Delta f_{1 / 2}$ $\left(\Delta f_{1 / 2}=f_{0} / Q_{L}\right)$ and increases strongly for lower offset frequencies. According to (1) the phase noise of the oscillator exhibits a $1 / f_{m}^{3}$ dependence in a certain range of the offset frequencies. The noise decreases strongly upon increasing loaded quality factor $Q_{L}$ of the resonator. The latter is strongly dependent on the microwave power.

The phase noise achieved for the oscillator was about $-24 \mathrm{dBc} / \mathrm{Hz}$ at the offset frequency of $10 \mathrm{~Hz}$. At this frequency the offset noise level is approximately the same as it was predicted by the Leeson model. However, above this frequency the noise level exceeds the predicted value (depicted in Fig. 4 by circles) calculated with the empirical relation by the Leeson model from the fit to the amplifier phase noise.

Therefore, not only the nonlinearity of the amplifier plays the crucial role for the oscillator circuit, but also the additional noise source resulted from the nonlinear response of the HTS resonator to the elevated microwave power.

The observed deviation of the phase noise can be explained by the current fluctuations in the HTS film resulting in extra phase noise due to the nonlinear properties of the HTS resonator. Higher order modes excite the third-order harmonic power and reduce the phase noise in the oscillator.

\section{CONCLUSION}

In conclusion, the nonlinear behavior of an HTS microstrip resonator with strongly coupled ports has been studied. The $2.3 \mathrm{GHz}$ oscillator was assembled on the base of the HTS resonator. The phase noise of the HEMT amplifier was found to be $-140 \mathrm{dBc} / \mathrm{Hz}$ at $1 \mathrm{kHz}$ frequency offset. This is transformed into an oscillator phase noise of $-78 \mathrm{dBc} / \mathrm{Hz}$ at $1 \mathrm{kHz}$ offset in the case of a cryogenic HTS resonator working at power $P=$ 
$12 \mathrm{dBm}$. The oscillator phase noise is attributed to the low $1 / f$ noise of the amplifier below $100 \mathrm{~Hz}$. For the frequency offset of above $100 \mathrm{~Hz}$ it also contains an additional noise coming from the nonlinear response of the HTS thin film to the $12 \mathrm{dBm}$ microwave power. Both noise contributions are present, though the port coupling was optimized in order to achieve the minimal phase noise level in the oscillator circuit. The obtained results can be useful for the analysis and design of the HTS-based microwave devices for satellite applications.

\section{ACKNOWLEDGMENT}

The authors would like to thank F. Ratzel for technical support.

\section{REFERENCES}

[1] M. Klauda, T. Kasser, B. Mayer, C. Neumann, F. Schnell, B. Aminov, A. Baumfalk, H. Chaloupka, S. Kolesov, H. Piel, N. Klein, S. Schornstein, and M. Bareiss, "Superconductors and cryogenics for future communication systems," IEEE Trans. Microw. Theory Tech., vol. 48, pp. 1227-1239, July 2000.
[2] Z. G. Galani, M. J. Bianchini, R. C. Waterman Jr, R. Dibiase, R. W. Laton, and J. B. Cole, "Analysis and design of a single-resonator GaAs FET oscillator with noise degeneration," IEEE Trans. Microw. Theory Tech., vol. 32, pp. 1556-1565, Dec. 1984.

[3] H. J. Siweris and B. Schiek, "Analysis of noise upconversion in microwave FET oscillators," IEEE Trans. Microw. Theory Tech., vol. 33, pp. 233-242, Mar. 1985.

[4] J. Geerk, A. Zaitsev, G. Linker, R. Aidam, R. Schneider, F. Ratzel, R. Fromknecht, B. Scheerer, H. Reiner, E. Gaganidze, and R. Schwab, "A 3-Chamber deposition system for the simultaneous double-sided coating of 5-inch wafers," IEEE Trans. Appl. Supercond., vol. 11, pp. 3856-3858, Mar. 2001

[5] R. Aidam, J. Geerk, G. Linker, F. Ratzel, J. Reiner, R. Schneider, R. Smithey, A. G. Zaitsev, E. Gaganidze, and R. Schwab, "Y-Ba-Cu-O thin films on 3" sapphire wafers for microwave devices," IEEE Trans Appl. Supercond., vol. 11, pp. 357-360, Mar. 2001.

[6] A. G. Zaitsev, R. Schneider, G. Linker, F. Ratzel, R. Smithey, and J. Geerk, "Nonlinear effects in $\mathrm{YBa}_{2} \mathrm{Cu}_{3} \mathrm{O}_{7-x}$ microstrip resonators on sapphire," Appl.Phys.Lett., vol. 79, pp. 4174-4176, Dec. 2001.

[7] D. E. Oates, P. P. Nguyen, G. Dresselhaus, M. S. Dresselhaus, C. W. Lam, and S. M. Ali, "Measurements and modeling of linear and nonlinear effects in striplines," J. Supercond., vol. 5, pp. 361-369, Aug. 1992

[8] J. C. Booth, L. R. Vale, R. H. Ono, and J. H. Claassen, "Power-dependent impedance of high temperature superconductor thin films: Relation to harmonic distortion," J. Supercond., vol. 14, pp. 65-79, Feb. 2001.

[9] D. B. Leeson, "A simple model of feedback oscillator noise spectrum," Proc. IEEE, vol. 54, pp. 329-330, 1966. 